\title{
A new sample of bright galaxy pairs in UZC ${ }^{\star}$ (Research Note)
}

\author{
P. Focardi ${ }^{1}$, V. Zitelli ${ }^{2}$, S. Marinoni ${ }^{2}$, and B. Kelm ${ }^{1}$ \\ 1 Dipartimento di Astronomia, Universitá di Bologna, Italy \\ e-mail: paola.focardi@unibo.it \\ 2 INAF - OABO, via Ranzani 1, 40127 Bologna, Italy \\ Received 21 March 2006 / Accepted 19 April 2006
}

ABSTRACT

\begin{abstract}
Aims. We present a new sample of bright galaxy pairs extracted applying an objective selection code to the UZC catalog. The sample is volume-limited to $M_{z w}=-18.9+5 \log h$ and contains 89 galaxy pairs.

Methods. We analyze the kinematical, morphological, and photometrical properties of galaxies belonging to this sample.

Results. We show that velocity separation, $\left|\Delta v_{\mathrm{r}}\right|$, between pair members is significantly lower in spiral type $(\mathrm{S}+\mathrm{S})$ pairs than in earlytype $(\mathrm{E}+\mathrm{E})$ and mixed $(\mathrm{E}+\mathrm{S})$ pairs. This indicates that truly isolated galaxy pairs are more likely to be found among $\mathrm{S}+\mathrm{S}$ pairs. We show that ellipticals are rare and underluminous in $\mathrm{B}$ and that late spirals $(T \geq 4)$ are overluminous.

Conclusions. We confirm that the formation of bright ellipticals is a phenomenon linked to group/cluster environment. while galaxygalaxy interaction may enhance the blue luminosity of disk galaxies through SF phenomena. This last statement is supported by the presence of strong FIR emission from early spirals in this sample and by the high frequency of the AGN/SB phenomenon, revealed mainly in pairs of low relative radial velocity separation showing signs of interaction.
\end{abstract}

Key words. galaxies: general - galaxies: fundamental parameters - galaxies: interactions

\section{Introduction}

It is well-known and accepted that starburst (SB) activity can be triggered, and possibly enhanced, by galaxy encounters. On a theoretical basis (Barnes \& Hernquist 1991), galaxy interaction is expected to redistribute large amounts of material towards the galaxy central regions, which may consequently trigger and fuel violent bursts of star formation. Galaxy collisions, eventually leading to a final merging, can produce strong alterations of galaxy morphologies and even originate complex structures, such as tidal tails and bridges. Instabilities in the discs, arising from tidal interactions, may induce formation of galaxy bars (Noguchi 1988; Barnes \& Hernquist 1991), which, generating an inflow of gas towards the galaxy central regions, might even activate AGN phenomenon. This theoretical scenario, however, is not adequately supported by observations. Even though, starting with Larson \& Tinsley (1978), there has been growing evidence, obtained from several different samples, of an increase of star formation in interacting galaxy systems (e.g., Kennicutt \& Keel 1984; Kennicutt 1987; Keel 1993, 1996; Donzelli \& Pastoriza 1997; Barton et al. 2000), a one-to-one correlation between galaxy-galaxy interaction and star formation remains unclear. In fact, only an extremely limited number of objects (ULIRGs, Sanders \& Mirabel 1996) show a fraction of interacting galaxies nearly close to $100 \%$ (Sanders et al. 1988; Borne et al. 1999), while there are several interacting systems showing no sign of star formation. The situation becomes even more complex and controversial for the so-called AGNinteraction paradigm for which conflicting results have been

* Table 1 is only available in electronic form at the CDS via anonymous ftp to cdsarc.u-strasbg.fr $(130.79 .128 .5)$ or via http://cdsweb.u-strasbg.fr/cgi-bin/qcat? J/A+A/456/467 given so far (Dahari 1985; Keel et al. 1985; Fuentes-Williams \& Stocke 1988; Rafanelli et al. 1995; MacKenty 1989; Kelm et al. 1998; De Robertis et al. 1998; Schmitt 2001; Kelm et al. 2004). However, these results relate to samples that are often small, have been selected by different methods and criteria, and may be biased towards or against certain kind of systems. A large part of controversy is thus likely to be ascribed to selection inhomogeneities among the samples.

Galaxy pairs are the ideal sites in which to investigate the role of a close companion on galaxy formation and evolution. So far, two large samples of "nearby" galaxy pairs are available: the Karactchensev sample (KPG, Karachentsev 1972) and its southern counterpart (RR, Reduzzi \& Rampazzo 1995). The first one has been selected by visual inspection of POSS plates, the second one has been extracted applying KPG criteria to the ESO-LV catalog (Lauberts \& Valentijn 1989).

Recent availability of large complete nearby 3D galaxy catalogs has made it possible to select galaxy samples having welldefined environment characteristics (Focardi \& Kelm 2002). We present here a new volume-limited sample of 89 bright galaxy pairs (UZC-BGP) selected applying an objective algorithm to the UZC catalog (Falco et al. 1999). This sample does not suffer from velocity/distance biases or contamination by projection effects, as the galaxies are already close in 3D space. In this paper, we present the UZC-BGP sample (Sect. 2) and, on the basis of presently available data, discuss its morphological content and show how it relates to the radial velocity separation $\left(\left|\Delta v_{\mathrm{r}}\right|\right)$ between galaxies in the pairs (Sect. 3). We analyze B luminosity and NIR colors (Sect. 4), FIR emission (Sect. 5), and the presence of nuclear activity (AGN/SB) (Sect. 6). The conclusions are drawn in Sect. 7. A Hubble constant of $H_{0}=100 h \mathrm{~km} \mathrm{~s}^{-1} \mathrm{Mpc}^{-1}$ is assumed throughout. 


\section{The sample}

The bright pair galaxy sample has been selected with an adapted version of the neighbor search algorithm of Focardi \& Kelm 2002. Each UZC galaxy having $M_{z w} \leq-18.9+5 \log h, v_{\mathrm{r}} \in$ [2500-7500] $\mathrm{km} \mathrm{s}^{-1}$, and $\left|b^{\mathrm{II}}\right| \geq 30^{\circ}$ has been explored on a surrounding area characterized by a projected radius $R_{\mathrm{p}}=$ $1 h^{-1} \mathrm{Mpc}$ and a radial velocity "distance" $\left|\Delta v_{\mathrm{r}}\right| \leq 1000 \mathrm{~km} \mathrm{~s}^{-1}$. The limit in $\left|b^{\mathrm{II}}\right|$ has been set to minimize the effects of galactic absorption. The lower limit in $v_{\mathrm{r}}$ reduces distance uncertainty due to peculiar motions and avoids contamination by the Virgo cluster. The upper limit in $v_{\mathrm{r}}$ results in the combination of UZC magnitude limit $\left(m_{z w} \leq 15.5\right)$ and our imposed limit in $M_{z w}$. The radial velocity "radius" $\left(\left|\Delta v_{\mathrm{r}}\right|\right)$ has been set equal to $1000 \mathrm{~km} \mathrm{~s}^{-1}$, which is large enough to not induce an artificial cut in the relative velocity of galaxies in pairs and to prevent contamination by galaxy groups. Galaxies having only one luminous $\left(M_{z w} \leq-18.9+5 \log h\right)$ neighbor within $r_{\mathrm{p}} \leq 200 h^{-1} \mathrm{kpc}$ and no other ones up to $R_{\mathrm{p}} \leq 1 h^{-1} \mathrm{Mpc}$ are part of the bright galaxy pair sample (UZC-BGP). The galaxy-galaxy projected distance radius $\left(r_{\mathrm{p}}\right)$ has been set equal to $200 \mathrm{~h}^{-1} \mathrm{kpc}$ to account for possible huge haloes tied to bright galaxies (Bahcall et al. 1995; Zaritsky et al. 1997). The large scale radius $\left(R_{\mathrm{p}}\right)$ has been set equal to $1 h^{-1} \mathrm{Mpc}$ to ensure the absence of luminous galaxies on the scale typical of groups.

Application of the above-described selection criteria has produced a sample of 89 galaxy pairs, listed in Table 1 (available at the CDS). For each galaxy, we give pair and galaxy identification (Cols. 1 and 2), equatorial coordinates $\mathrm{RA}_{\mathrm{J} 2000}$, and $\mathrm{Dec}_{\mathrm{J} 2000}$ (Cols. 3 and 4), apparent magnitude $m_{z w}$ and radial velocity $v_{\mathrm{r}}$ from UZC (Cols. 5 and 6), and morphological classification from LEDA (Col. 7). For 18 galaxies, morphological classification is not too accurate, thus it is indicated by an $\mathrm{E}$ or a $\mathrm{S}$ followed by a question mark. The presence of a ring is denoted by $\mathrm{Y}$ (yes) in Col. 8, and the presence of nuclear activity, from available literature data (NED), is indicated in Col. 9. Column 10 indicates if the galaxy has already been identified as a member of either a galaxy pair or a compact group.

In eleven cases, and as consequence of the imposed limit in absolute magnitude, the algorithm has detected only the most luminous galaxies of a compact group (HCG, Hickson 1982; Hickson et al. 1992; KTG, Karachentseva et al. 1979; UZC-CG, Focardi \& Kelm 2002). For the same reason, only the brightest galaxies of 6 known pairs (KPG) have been detected. In these last cases, however, the algorithm has detected a bright companion that KPG visually based criteria failed to identify. All previously identified galaxy pairs (19) fulfilling our selection requirements have been detected by the algorithm. The remaining 53 pairs are new (not identified before).

Having applied well-defined selection criteria to a large and complete galaxy catalog we can compute the fraction of galaxies in pairs, which turns out to be $6 \%$. Luminous galaxies are rare, as the vast majority (88\%) of UZC-BGP has $M_{z w}-5 \log h>$ -20. Thus, galaxy pairs do not appear ideal sites in which bright galaxies can originate, contrary to what seems to be the case for galaxy groups, especially for elliptical dominated ones (Kelm \& Focardi 2004). The magnitude difference $\left(\left|\Delta M_{z w}\right|\right)$ between pair members is less than 0.5 for the majority $(76 \%)$ of galaxies. Most pairs (72\%), display a $\left|\Delta v_{\mathrm{r}}\right| \leq 200 \mathrm{~km} \mathrm{~s}^{-1}$, while more than half the pairs $(64 \%)$ have $r_{\mathrm{p}}<100 h^{-1} \mathrm{kpc}$.

\section{Morphological content of the sample}

Morphological classification is available (LEDA) for 164 galaxies $(92 \%$ of the total sample); 79 pairs possess classification
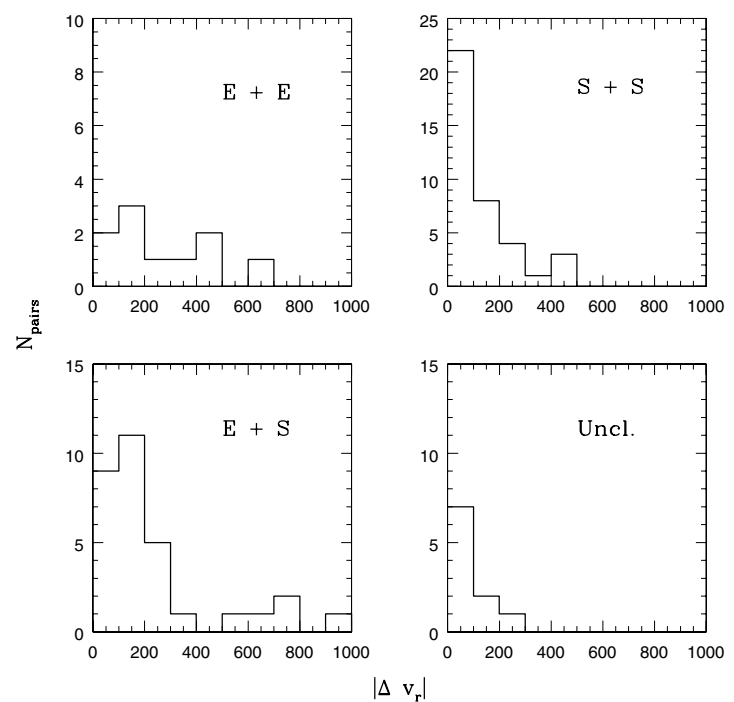

Fig. 1. The radial velocity separation, for $\mathrm{E}+\mathrm{E}$ (upper left), $\mathrm{S}+\mathrm{S}$ (upper right), $\mathrm{E}+\mathrm{S}$ (lower left), and unclassified (lower right) pairs.

for both members, 6 for only one, and 4 lack this information for both members. As LEDA also provides the morphological code $(T)$, we have used it to classify the 79 UZC-BGP pairs with morphological classifications. Of these, 10 are E+E pairs (composed of early-type galaxies only, both with $T<0$ ), 38 are $\mathrm{S}+\mathrm{S}$ (composed of spirals only, both with $T \geq 0$ ), and 31 are $\mathrm{E}+\mathrm{S}$ pairs (one galaxy with $T<0$ and the other with $T \geq 0$ ). This gives a morphological content of our sample of $13 \% \mathrm{E}+\mathrm{E}, 48 \% \mathrm{~S}+\mathrm{S}$, and $39 \% \mathrm{E}+\mathrm{S}$, compared to the corresponding KPG figures, which are $14 \%, 60 \%$, and $26 \%$, respectively. While early-type pair content of UZC-BGP matches the KPG one extremely well, the fraction of mixed and spiral type pairs are rather different, given that UZC-BGP has a higher content of $\mathrm{E}+\mathrm{S}$ (and consequently a lower content of $\mathrm{S}+\mathrm{S}$ ) than the KPG sample. We stress, however, that $8 \mathrm{E}+\mathrm{S}$ UZC-BGP contain either a SB0 or a S0/a galaxy, which (according to its $T$ value just below 0 ) has been counted as an early-type galaxy. Actually, these galaxies are border line objects and including them among early-type or spirals is somewhat arbitrary. Had we adopted a less conservative criterion and included those pairs in the $\mathrm{S}+\mathrm{S}$ ones, it would have changed the fraction of UZC-BGP $\mathrm{S}+\mathrm{S}$ and $\mathrm{E}+\mathrm{S}$ pairs to $58 \%$ and $29 \%$ respectively in much better agreement with KPG morphological content. No significant difference emerges either in magnitude or in projected distance when the morphological composition of the pairs is taken into account. The radial velocity separation instead appears to be significantly different (Fig. 1). S+S pairs display a narrower distribution than the $\mathrm{E}+\mathrm{S}$ and $\mathrm{E}+\mathrm{E}$ ones, while curiously unclassified pairs distribute similarly to $\mathrm{S}+\mathrm{S}$. A Kolmogornov Smirnov test confirms the difference ( $99.3 \%$ c.l.) in the $\left|\Delta v_{\mathrm{r}}\right|$ distribution of $\mathrm{E}+\mathrm{E}$ and $\mathrm{S}+\mathrm{S}$ pairs and of $\mathrm{S}+\mathrm{S}$ and $\mathrm{E}+\mathrm{S}$ pairs (99.5\% c.l.). Also, the $\left|\Delta v_{\mathrm{r}}\right|$ distribution of unclassified pairs is significantly different from the one of $\mathrm{E}+\mathrm{E}$ and $\mathrm{E}+\mathrm{S}$ (99.2\% c.l. in both cases). The wider radial velocity separation of $\mathrm{E}+\mathrm{E}$ and $\mathrm{E}+\mathrm{S}$ might indicate the presence of a deeper potential well, implying that a significant fraction of these pairs could be part of larger bound structures. If this were the case, truly isolated galaxy pairs would be more likely to be found among $\mathrm{S}+\mathrm{S}$ pairs only.

Figure 2 shows the morphological type code $(T)$ distribution of galaxies in the 79 UZC-BGP pairs having classification for both members (upper left panel). The other panels show morphological content of E+E (upper right), E+S (lower left), 

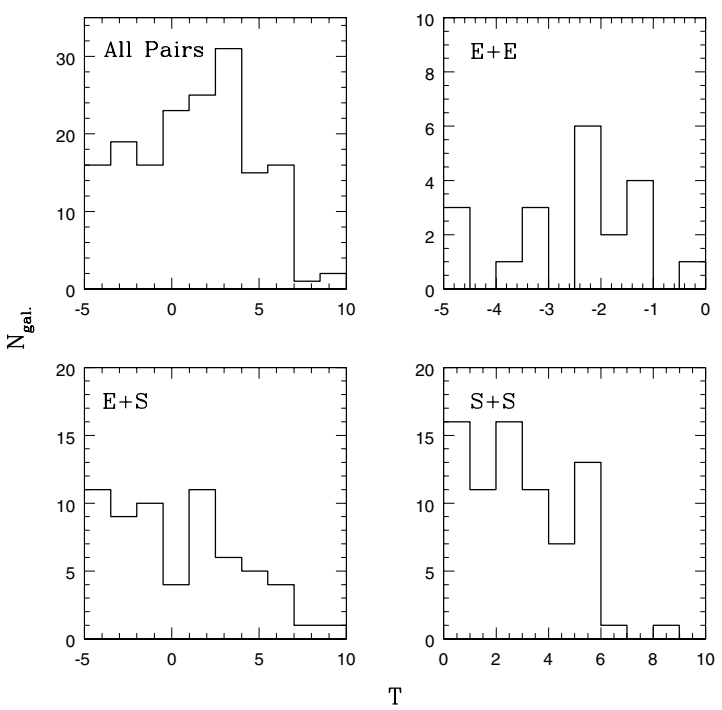

Fig. 2. Morphological content of the 79 UZC-BGP pairs having classification for both members (upper left). The other panels show the content of the $10 \mathrm{E}+\mathrm{E}, 31 \mathrm{E}+\mathrm{S}$, and $38 \mathrm{~S}+\mathrm{S}$ pairs.

and $S+S$ (lower right) pairs. Figure 2 (upper left) illustrates that UZC-BGP sample is dominated by early spirals $(0 \leq T<4)$. Late spirals $(T \geq 4)$ are much rarer, while early-type galaxies $(T<0)$ are quite abundant. The upper right panel of Fig. 2 also shows that ellipticals $(T<-3)$ in early-type pairs are not as frequent as S0s $(-3 \leq T<0)$, but the low statistic does not allow us to draw a definitive conclusion on this point.

\section{Luminosity and colors of BGP member galaxies}

We have collected (from NED) optical and NIR photometric data for UZC-BGP galaxies. $B$ magnitude is available for 128 galaxies and J H K (2-Mass) magnitudes for 168 galaxies (respectively, $72 \%$ and $94 \%$ of the total sample). $V$ magnitude is available only for $21 \%$ of the sample and will not be used.

Figure 3 shows the $M_{B}$ distribution of E and S0s (upper panels) and early and late spirals (lower panels) in pairs of different morphology. The shaded distribution always refers to minority populations, which are $\mathrm{E}+\mathrm{E}$ pairs for early-type galaxies (upper panels) and $\mathrm{E}+\mathrm{S}$ for spirals (lower panels). The solid distribution relates to $\mathrm{E}+\mathrm{S}$ pairs (upper panels) and to $\mathrm{S}+\mathrm{S}$ pairs (lower panels). The upper left panel of Fig. 3 shows that ellipticals are underluminous in $\mathrm{B}$, a tendency that appears especially strong when these galaxies are members of E+E pairs. On the other hand, spiral galaxies show a luminosity distribution that extends at higher values. This is particularly true for late spirals (lower right panel). Galaxy pairs thus appear to be characterized by a population of "underluminous" ellipticals and of "overbright" late spirals, implying that this kind of environment might disfavor the formation of bright early-type galaxies and favor the luminosity increase of late spirals. The latter phenomenon is probably linked to a SF enhancement induced by interaction processes. No significant differences are found among spiral galaxies in different kinds of pairs even though the lower left panel of Fig. 3 indicates that the early spirals of higher luminosity are found in $\mathrm{S}+\mathrm{S}$ pairs. This might indicate an enhancement in luminosity due to interaction phenomena occurring mainly in $\mathrm{S}+\mathrm{S}$ pairs.

Figure 4 shows the color magnitude diagram $\left(B-K\right.$ vs. $\left.M_{K}\right)$ of E and S0s (upper panels) and early and late spirals (lower panels). Symbols are filled when galaxy morphology agrees with
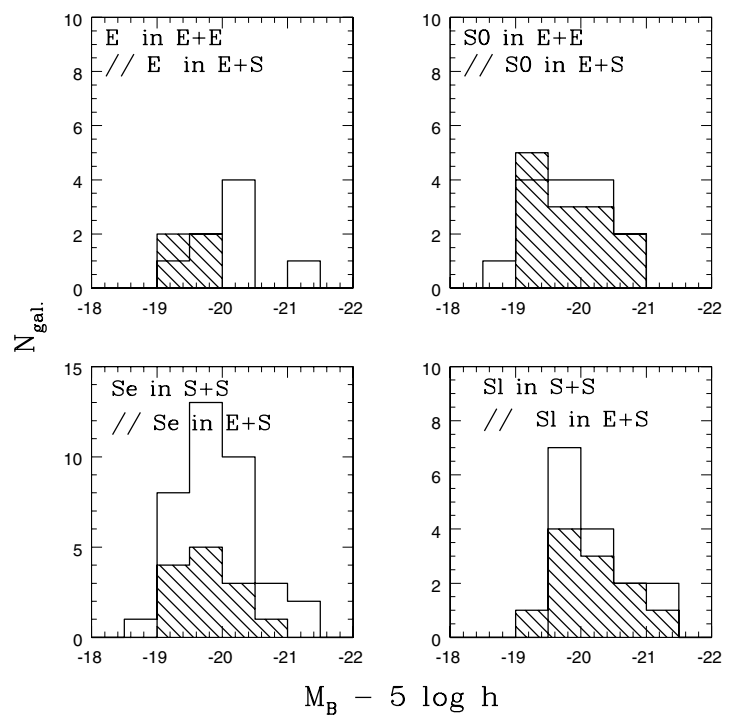

Fig. 3. $M_{B}$ distribution of E (upper left), S0s (upper right), early (lower left), and late (lower right) spirals. The dashed histograms represent the $M_{B}$ distribution of galaxies belonging to minority population, i.e., $\mathrm{E}+\mathrm{E}$ in the upper panels, $\mathrm{E}+\mathrm{S}$ in the lower ones.
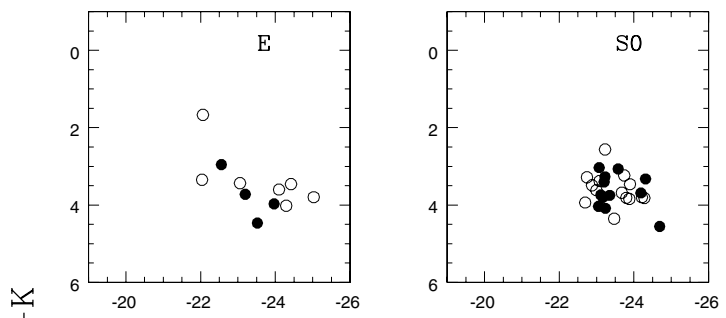

!
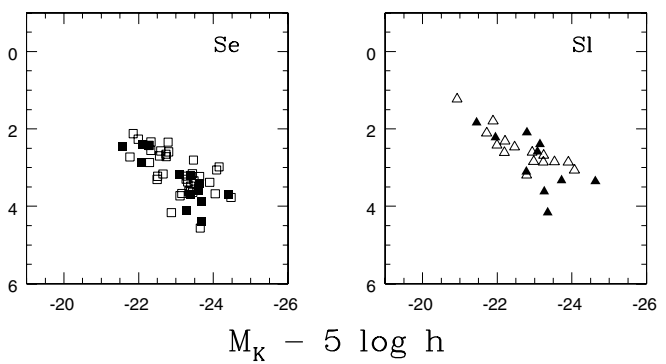

Fig. 4. $B-K$ versus $M_{K}$ distribution. The upper panels show the distribution of $\mathrm{E}$ and $\mathrm{S} 0$ in $\mathrm{E}+\mathrm{E}$ (filled circles) and $\mathrm{E}+\mathrm{S}$ pairs (empty circles). The lower panels show the distribution of early and late spirals; filled symbols indicate spirals in $\mathrm{S}+\mathrm{S}$, while open symbols spirals in $\mathrm{E}+\mathrm{S}$ pairs.

pair one, and they are not filled when it does not (i.e., open symbols always represent $\mathrm{E}+\mathrm{S}$ pairs, filled circles in the upper panels represent $\mathrm{E}+\mathrm{E}$ pairs, filled symbols in the lower ones represent $\mathrm{S}+\mathrm{S}$ pairs). Spirals (lower panels) distribute in the usual way, the brightest in $\mathrm{K}$ being the reddest. Late spirals are significantly bluer than early spirals. This happens as a consequence of the B luminosity distribution of late spirals, which is shifted by about one magnitude with respect to that of early spirals (Fig. 4). Early-type galaxies are bluer than normal $\left(\langle B-K\rangle_{E-S 0} \sim 4.5\right)$. S0s (upper right panel) appear particularly concentrated, while the sequence of ellipticals (upper left panel) is much extended. The $\mathrm{K}$ brightest ellipticals in $\mathrm{E}+\mathrm{S}$ pairs (open circles) deviate from the normal sequence by being bluer than expected. This happens as a consequence of their also being the brightest in $M_{B}$ (see Fig. 3). We thus find that ellipticals in pairs display 

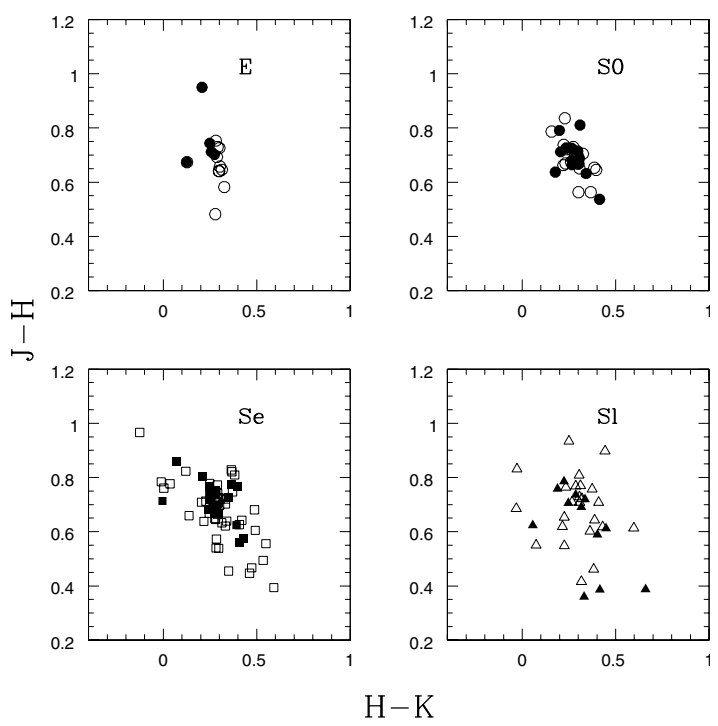

Fig. 5. $J-H$ versus $H-K$ distribution. The upper panels show the distribution of $\mathrm{E}$ and $\mathrm{S} 0$ in $\mathrm{E}+\mathrm{E}$ (filled circles) and $\mathrm{E}+\mathrm{S}$ pairs (empty circles). The lower panels the distribution of early and late spirals, filled symbols indicate spirals in $\mathrm{S}+\mathrm{S}$, open symbols spirals in $\mathrm{E}+\mathrm{S}$ pairs.

anomalous characteristics, which appear to relate to pair morphology: ellipticals in $\mathrm{E}+\mathrm{E}$ are underluminous in B (see Fig. 3), while ellipticals of higher B luminosity, in $\mathrm{E}+\mathrm{S}$ pairs, are bluer than expected (Fig. 4). This finding suggests that (although limited) interaction phenomena affecting ellipticals in pairs are more likely to occur in $\mathrm{E}+\mathrm{S}$ than in $\mathrm{E}+\mathrm{E}$, which appear to host normal low luminosity ellipticals.

Figure 5 shows the NIR color-color $(J-H$ vs. $H-K)$ diagram for E, S0, early, and late spirals in pairs whose morphology is either accordant (filled symbols) or discordant (open symbols) with the galaxy one. The upper left panel confirms that ellipticals in pairs have bluer than normal colors. In fact, all but one (belonging to an $\mathrm{E}+\mathrm{E}$ pair) are bluer than $J-H=$ 0.78 , which is the normal value for "ordinary" (bulge dominated) galaxies (Glass 1984), and all but two (both belonging to E+E pairs), have $H-K$ larger than 0.22 , which is again the normal value for bulge dominated galaxies (Glass 1984). The distribution of spirals (lower panels) is significantly shifted towards bluer $J-H$ and redder $H-K$ colors. Most galaxies have $H-K>0.26$, which is typical of emission line galaxies (Glass 1984; Glass \& Morwood 1985), and the distribution of late spirals (lower right panel) is much more scattered (in $H-K$ ) than the distribution of early spirals (lower left panel).

\section{Far Infrared emission from UZC-BGP}

We have collected IRAS data (from NED) for all galaxies in our sample. As expected, the fraction of FIR emitting galaxies increases as the morphological type advances. Table 2 (Col. 2) shows the morphological content, in ellipticals, S0s, and early and late spirals, of the UZC-BGP sample, and the corresponding number of galaxies having FIR emission measured in all 4 IRAS bands (Col. 3). In Col. 4 the number of galaxies having measured $f_{60}$ and $f_{100}$ and an upper limit estimate for $f_{12}$ and/or $f_{25}$ is indicated. In Col. 5 the number of galaxies having only upper limit estimates either for all the 4 bands or for at least one of the shortest $\left(f_{12}, f_{25}\right)$ and of the longest $\left(f_{60}, f_{100}\right)$ wavelength bands is listed.

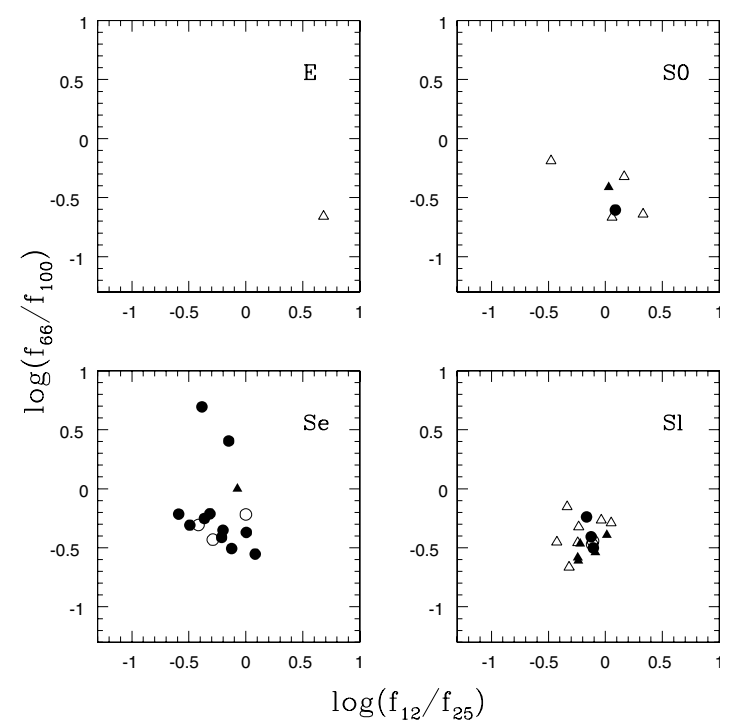

Fig. 6. IRAS color color diagram, for ellipticals (upper left), S0s (upper right), early (lower left), and late (lower right) spirals. Symbols are either filled or unfilled in analogy with those adopted in Figs. 4 and 5. Circles stand for galaxies having FIR emission measured in all 4 IRAS bands, triangles for galaxies having only $f_{60}$ and $f_{100}$ measured, and an upper limit estimate for either both, or at least one, of $f_{12}$ and $f_{25}$.

Table 2. IRAS emission from UZC-BGP galaxies.

\begin{tabular}{lcccc}
\hline \hline Morphological Code & $N_{\text {Tot }}$ & $N_{\text {IRAS }}$ & $N_{60-100}$ & $N_{\text {ul }}$ \\
\hline$T<-3$ & 18 & 0 & 1 & 3 \\
$-3 \leq T<0$ & 35 & 1 & 6 & 4 \\
$0 \leq T<4$ & 72 & 14 & 13 & 6 \\
$T \geq 4$ & 39 & 6 & 13 & 4 \\
\hline
\end{tabular}

The fraction of IRAS detected galaxies turns out to be $22 \%$ (4/18) for ellipticals, $31 \%$ (11/35) for S0s, 46\% (33/72) for early spirals, and 59\% (23/39) for late spirals, which is rather high (especially for early-type galaxies). These fractions decrease to $5 \%, 20 \%, 38 \%$, and $49 \%$, considering only galaxies for which it is possible to compute $L_{\text {FIR }}$ (Cols. 3 and 4 of Table 2). However, the computed fractions must be considered lower limit estimates of the real ones, as faint galaxies easily go undetected in a flux limited survey such as IRAS. If we keep UZC-BGP galaxies up to $m_{z w} \leq 14.5$ and recompute the fractions, we find that galaxies detected and having measurable $L_{\mathrm{FIR}}$ are $40 \%$ and $10 \%$ for E, $47 \%$ and $27 \%$ for S0s, $62 \%$ and $53 \%$ for early spirals, and $79 \%$ and $64 \%$ for late spirals.

Figure 6 shows the IRAS color color diagram for ellipticals (upper left panel), S0s (upper right), early (lower left), and late (lower right) spirals. Circles represent galaxies for which FIR emission has been measured in all 4 IRAS bands. In analogy with Figs. 5 and 6 , they are filled if the galaxy resides in an accordant morphology pair ( $\mathrm{E}+\mathrm{E}$ for the upper panels, $\mathrm{S}+\mathrm{S}$ for the lower ones) and they are unfilled if the galaxy resides in an $\mathrm{E}+\mathrm{S}$ pair. Triangles represent galaxies for which both $f_{60}$ and $f_{100}$ have been measured (while an upper limit estimate of either both or at least one of $f_{12}$ and $f_{25}$ is available). They may be either filled or unfilled according to the above stated definition. The high number of filled circles in the lower left panel confirms that galaxy-galaxy interaction, occurring mainly in $\mathrm{S}+\mathrm{S}$ pairs, enhances FIR emission in early spirals. More than 
half of the early spirals lay above $\log \left(f_{60} / f_{100}\right)>-0.3$, suggesting the presence of SF phenomena (Sanders \& Mirabel 1996; Kim \& Sanders 1998). The two early spirals displaying the highest value of $f_{60} / f_{100}$ are NGC 7771, which is a known SB and NGC 5908. They belong to pairs in which both members emit in FIR. Late spirals show a much concentrated distribution and do not appear to "prefer" $\mathrm{S}+\mathrm{S}$ pairs to $\mathrm{E}+\mathrm{S}$ ones. In Fig. 6, we have not displayed galaxies that have an upper limit estimate of the flux in at least one of both the short and the long IRAS wavelength bands. If we also take into account these galaxies, we find that FIR emission is detected in only 3 early-type galaxies, belonging to 3 distinct $E+E$ pairs, in $22 \mathrm{E}+\mathrm{S}$ pairs (5 of which show emission for both members), and in $27 \mathrm{~S}+\mathrm{S}$ pairs (9 of which show emission for both members). This gives a frequency of FIR emission of $30 \%$ for $\mathrm{E}+\mathrm{E}$ and $69 \%$ for both $\mathrm{E}+\mathrm{S}$ and $\mathrm{S}+\mathrm{S}$. There is no significative difference in the distribution of $\left|\Delta v_{\mathrm{r}}\right|$ and $r_{\mathrm{p}}$ between UZC-BGP hosting and non-hosting one (or two) IRAS sources, even though the former show a narrower $\left|\Delta v_{\mathrm{r}}\right|$ distribution than the latter.

We have computed Far Infrared Luminosity $\left(L_{\mathrm{FIR}}\right)$ (following Sanders \& Mirabel 1996) for galaxies in which $f_{60}$ and $f_{100}$ have been measured, and compared it to $L_{B}$. Most galaxies follow the $\log \left(L_{\mathrm{FIR}}\right)-\log \left(L_{B}\right)$ relation, only 3 early-type galaxies deviate significantly from it, and of these the one showing the lowest FIR and $B$ luminosity is the only FIR emitting elliptical. The two strongest FIR sources are the brightest in B early spirals (Fig. 4, lower left panel). No LIRGs are present.

\section{Nuclear activity in UZC-BGP}

We have inspected available data (NED) looking for the presence of nuclear activity in UZC-BGP. This information is provided in Col. 9 of Table 1 . The sample contains 10 SB galaxies, 4 Liners, 3 Sy2, 1 Sy1, and 2 AGN. (In the case of a double classification, e.g. Sy2/SB, we have retained the first one). Adding together all kind of activities, we find that the incidence of nuclear activity phenomenon in UZC-BGP galaxies is $11 \%$, which is quite high considering that it must be taken as a lower limit estimate. SB activity appears to be the most frequent one.

Four pairs have both members active: BGP 1, BGP 85, BGP 87, and BGP 89. Three of these pairs are E+S and one (BGP 89) is a $\mathrm{S}+\mathrm{S}$. The pairs hosting only one active member are $3 \mathrm{E}+\mathrm{S}, 8 \mathrm{~S}+\mathrm{S}$, and 1 unclassified one. In all, but three cases (BGP 73A, BGP 80B, and BGP 82A), active galaxies are first ranked (or of equal luminosity) in the pair. The vast majority of active galaxies are spirals, which is not surprising due to the large incidence of SB activity in this sample. Only 3 are early-type, namely NGC 6251 (BGP 79A), which is an elliptical, NGC 7550 (BGP 85A, E/S0), and NGC 7679 (BGP 87A, SB0/a). All of them are members of $\mathrm{E}+\mathrm{S}$ pairs. Half of the active galaxies have $M_{z w} \leq-19.5-5 \log h$ and are thus among the brightest in the sample. The most luminous active galaxy is the SB NGC 23 (BGP 2A) with $M_{z w}-5 \log h=$ -20.8 . The three Sy 2 galaxies have normal/high luminosities $\left(-20.3 \leq M_{z w}-5 \log h \leq-19.3\right)$, the only Sy 1 detected has $M_{z w}-5 \log h=-20.4$. The radial velocity separation $\left|\Delta v_{\mathrm{r}}\right|$ of $14 / 16$ pairs with one (or both) active members is less than $200 \mathrm{~km} \mathrm{~s}^{-1}$. The two pairs exceding this limit are BGP 79 (hosting a Sy 2) and BGP 85 (hosting two AGNs). If nuclear activity is related to galaxy-galaxy interaction, pairs having $\left|\Delta v_{\mathrm{r}}\right| \leq$ $200 \mathrm{~km} \mathrm{~s}^{-1}$ appear as the ones in which this phenomenon is most likely to occur.

We have inspected the DSS images of UZC-BGP hosting one (or both) active member(s) to look for signs of morphological distorsion and interaction. Strong interaction occurs in all 4 UZC-BGP pairs with both members active, namely the two Liners in BGP 1 that are part of a well-known system of interacting galaxies (VV 254, the Taffy system), the two AGNs in BGP 85 (also part of HCG93), the Sy1/SB and Sy2 in BGP 87 (VV 329), and the SB/Liner and SB in BGP 89. Interaction between pair members is also present in BGP 7 and BGP 14, both hosting a SB galaxy. BGP 28A (Liner) and 38A (SB) are mergers, the latter in a less advanced stage. BGP 73A (SB/Sy1), $80 \mathrm{~B}(\mathrm{SB})$, and 83A (Sy2/SB) show signs of morphological distorsion. While the most luminous active galaxy of the sample (NGC 23 SB in BGP 2) has a large bar, but not visible interaction, the Liner in BGP 3, the SB in BGP 68, the Sy2/Radiogalaxy in BGP 79, and the SB in BGP 82 show no signs of distorsion. Thus, $15 / 20$ active galaxies in UZC-BGP show signs of interaction/distorsion providing support to the interaction activity scenario.

\section{Conclusions}

We have presented a new volume-limited sample of bright galaxy pairs selected from the UZC catalog by means of an objective neighbor-search algorithm. The sample contains 89 pairs whose members have a mutual projected distance $r_{\mathrm{p}} \leq$ $200 h^{-1} \mathrm{kpc}$ and no other bright $\left(M_{z w} \leq-18.9+5 \log h\right)$ galaxies within $R_{\mathrm{p}} \leq 1 h^{-1} \mathrm{Mpc}$ and $\left|\Delta v_{\mathrm{r}}\right| \leq 1000 \mathrm{~km} \mathrm{~s}^{-1}$.

We have shown that elliptical galaxies are rare $(10 \%)$ in pairs and underluminous in $\mathrm{B}$. The last characteristic holds especially for $\mathrm{E}$ in $\mathrm{E}+\mathrm{E}$ pairs $\left(M_{B}<-20+5 \log h\right)$, confirming previous findings (Kelm \& Focardi 2004) relating the formation of bright ellipticals to the phenomenon linked to the formation of group size systems. Analysis of NIR data provide evidence that ellipticals in E+S pairs are "bluer" (in $J-H$ and in $B-K$ ) than expected. Thus, for elliptical galaxies, the effect of a close bright companion appears to relate to its morphology having the maximum (even if limited) effect if it is a spiral. Curiously, in the $B-K$ vs. $M_{K}$ plot, S0 galaxies appear extremely concentrated, suggesting that S0s in pairs might constitute a population of galaxies with well-defined and unique characteristics.

Spiral galaxies, particularly late spirals, have their B luminosities enhanced, and we suggest that this may be due to galaxy-galaxy interaction phenomena, occurring mainly in $\mathrm{S}+\mathrm{S}$ pairs. These pairs display the narrowest radial velocity separation between members, a characteristic that make them appear to be the best candidates of truly isolated galaxy pairs.

Analyzing FIR (IRAS) emission, we have shown that it is particularly strong for early spirals, which are the galaxies of the highest FIR luminosity, even though none of them reaches the LIRG level. More than half of the early spirals detected by IRAS display flux ratio $\left(f_{60} / f_{100}\right)$ values that are typical of SF galaxies. The two early spirals that display the larger flux ratio are part of $\mathrm{S}+\mathrm{S}$ pairs, with both members FIR emitting and having radial velocity separation between the members well below $200 \mathrm{~km} \mathrm{~s}^{-1}$.

Finally, on the basis of available data, we have analyzed the occurrence of the AGN/SB phenomenon, which amounts to $11 \%$ (computed on the total number of galaxies), which we have shown is found mainly $(88 \%)$ in galaxy pairs having $\left|\Delta v_{\mathrm{r}}\right| \leq$ $200 \mathrm{~km} \mathrm{~s}^{-1}$, most $(78 \%)$ of which show signs of interaction. Detailed homogeneous spectroscopic data (Marinoni et al., in preparation) coupled with surface photometry (Zitelli et al., in preparation) will allow us to draw definitive conclusions on this topic. 
Acknowledgements. This work was supported by MIUR. S.M. acknowledges a fellowship by INAF-OAB. This research has made use of the NASA/IPAC Extragalactic Database (NED) and of the Lyon-Meudon Extragalactic Database (LEDA).

\section{References}

Bahcall, N. A., Lubin, J. M., \& Dorman, V. 1995, ApJ, 447, L81 Barnes, J. E., \& Hernquist, L. E. 1991, ApJ, 370, 65

Barton, E. J., Geller, M. J., \& Kenyon, S. J. 2000, ApJ, 530, 660

Borne, K. D., Bushouse, H., Colina, L., et al. 1999, Ap\&SS, 266, 137

Dahari, O. 1985, ApJS, 57, 643

De Robertis, M. M., Hayhoe, K., \& Yee, H. K. C. 1998, ApJS, 115, 163

Donzelli, C., \& Pastoriza, M. G. 1997, ApJS, 111, 181

Falco, E. E., Kurtz, M. J., Geller, M. J., et al. 1999, PASP, 111, 438

Focardi, P., \& Kelm, B. 2002, A\&A, 391, 35

Fuentes-Williams, T., \& Stocke, J. T. 1988, AJ, 96, 1235

Glass, I. S. 1984, MNRAS, 211, 461

Glass, I. S., \& Morwood, A. F. M. 1985, MNRAS, 214, 429

Hickson, P. 1982, ApJ, 255, 382

Hickson, P., Mendes de Oliveira C., Huchra, J. P., \& Palumbo, G. G. C. 1992, ApJ, 399, 353
Karachentsev, I. D. 1972, in Catalogue of Isolated Pairs in the Northern Emisphere, Comm. Spec. Ap. Obs., 7, 1 (KPG)

Karachentseva, V. E., Karachentsev, I. D., \& Shcherbanovskii, A. L. 1979, Astrofizicheskie Issledovaniia, 11, 3 (KTG)

Keel, W. C., Kennicutt, R., Hummel, E., \& van der Hulst, J. 1985, AJ, 90, 708

Keel, W. C. 1993, AJ, 106, 1771

Keel, W. C. 1996, AJ, 111, 696

Kelm, B., Focardi, P., \& Palumbo, G. G. C. 1998, A\&A, 335, 912

Kelm, B., Focardi, P., \& Zitelli, V. 2004, A\&A, 418, 25

Kelm, B., \& Focardi, P. 2004, A\&A, 418, 937

Kennicutt, R. C. Jr., \& Keel, W. C. 1984, ApJ, 279, L5

Kennicut, R. C. Jr. 1987, ApJ, 319, 61

Kim, D. C., \& Sanders, D. B. 1998, ApJS, 119, 41

Larson, R. B., \& Tinsley, B. M. 1978, ApJ, 219, 46L

Lauberts, A., \& Valentijn, E. 1989, The Surface Photometry Catalogue of the ESO-Uppsala Galaxies, ESO, Garching bei Munchen (ESO-LV)

MacKenty, J. W. 1989, ApJ, 343, 125

Noguchi, M. 1988, A\&A, 203, 259

Rafanelli, P., Violato, M., \& Baruffolo, A. 1995, AJ, 109, 1546

Reduzzi, L., \& Rampazzo, R. 1995, ApJL \& Comm., 30, 1 (RR)

Sanders, D. B., \& Mirabel, I. F. 1996, ARA\&A, 34, 749

Sanders, D. B., Soifer, B. T., Elias, J. H., et al. 1988, ApJ, 325, 74

Schmitt, H. R. 2001, AJ, 122, 2243

Zaritsky, D., Smith, R., Frenk, C., \& White, S. D. M. 1997, ApJ, 478, 39 